\title{
Mineralöl in Lebensmitteln - ein wunder Punkt der Kreislaufwirtschaft
}

\author{
Jochen Flasbarth
}

Published online: 7 May 2013

(C) Bundesamt für Verbraucherschutz und Lebensmittelsicherheit (BVL) 2013

Liebe Leserinnen, liebe Leser,

das Thema Migration von kritischen Mineralölbestandteilen aus Verpackungen in Lebensmittel bleibt aktuell. Untersuchungen des Kantonalen Labors Zürich vom Herbst 2009 hatten ergeben, dass Tageszeitungen durchschnittlich ca. 3000 mg Mineralöl pro kg Tageszeitung und daraus hergestellte Lebensmittelkartonverpackungen $300-1000 \mathrm{mg}$ Mineralöl pro kg Verpackungskarton enthalten. Diese kritischen Mineralölbestandteile setzen sich aus zwei Fraktionen zusammen:

- paraffinartige und naphthenartige Kohlenwasserstoffe - „mineral oil saturated hydrocarbons“ (MOSH), <24 C-Atome und

- aromatische Kohlenwasserstoffe - „mineral oil aromatic hydrocarbons“ (MOAH), die vor allem aus 1- bis 4-Ring-Systemen bestehen, größtenteils hoch alkyliert.

Diese Stoffe können über die Gasphase - teilweise auch durch Zwischenverpackungen hindurch - auf Lebensmittel übergehen. Somit können unbedruckte Verpackungen aus Recyclingkarton um den Faktor 10 höhere Mineralölgehalte als Frischfaserkartonverpackungen aufweisen. Die erhöhte Mineralölbelastung in den Recyclingkartons ist im Wesentlichen auf das Recycling von Zeitungspapier und die darin enthaltene Druckfarbe zurückzuführen. Auch in Deutschland

J. Flasbarth (ه)

Umweltbundesamt, Wörlitzer Platz 1,

06844 Dessau-Roßlau, Deutschland

e-mail: jochen.flasbarth@uba.de wurden Gehalte an Mineralöl in verschiedenen Lebensmitteln gefunden, die deutlich über den tolerierbaren Mengen liegen (BMELV 2012).

Für die Bewertung des Vorkommens von MOSH in Lebensmitteln steht gegenwärtig kein toxikologisch begründeter Grenzwert zur Verfügung. Der vom Joint FAO/WHO Expert Committee on Food Additives (JECFA) für MOSH mit niedriger bis mittlerer Viskosität (Class I und Class II) abgeleitete temporäre Wert für die maximal akzeptable tägliche Aufnahmemenge (ADI) von 0,01 mg/kg Köpergewicht wurde im Juni 2012 zurückgezogen, da auch neue toxikologische Daten die bestehenden Unsicherheiten in der gesundheitlichen Bewertung nicht ausräumen konnten.

Auch eine toxikologische Bewertung der MOAHFraktion ist auf Grund fehlender experimenteller toxikologischer Untersuchungen nicht möglich. Nur wenige Einzelsubstanzen sind untersucht. Jedoch ist davon auszugehen, dass auf Grund von Strukturhomologien zu polyzyklischen aromatischen Kohlenwasserstoffen (PAK) in der komplexen Mischung ein kanzerogenes Potential vorhanden ist. Da bei genotoxischen Kanzerogenen kein Schwellenwert für die toxikologische Wirkung abgeleitet werden kann, muss die Exposition des Verbrauchers mit der MOAHFraktion aus Mineralölen soweit wie möglich gesenkt werden (BfR 2009).

Eine Zwischenlösung können etwa Innenbeutel aus geeignetem Material (Aluminium oder PETFolien) oder spezielle Barriereschichten sein. An der Weiterentwicklung solcher Lösungen arbeiten die Verpackungshersteller intensiv. Seitens des Bundesministeriums für Ernährung, Landwirtschaft und Verbraucherschutz werden Entwürfe für zwei Verordnungen zur Änderung der Bedarfsgegen- 
ständeverordnung erarbeitet, das Bundesinstitut für Risikobewertung arbeitet an der Optimierung der Messmethoden. Damit werden neue Rahmenbedingungen für eine sicherere Lebensmittelverpackung gesetzt.

Für eine nachhaltige Lösung auch im Sinne einer funktionierenden Kreislaufwirtschaft und damit des Ressourcenschutzes ist es jedoch wichtig, dass alle Akteure in der Wertschöpfungskette ihren Beitrag zur Verringerung der Einträge in den Papierkreislauf leisten. Verpackungen aus recyceltem Altpapier sind im Vergleich zu Verpackungen aus Frischfasern oder mit zusätzlichen Zwischenverpackungen aus Aluminium oder PET-Folien als umweltfreundlicher und ökologisch sinnvoller einzustufen. Der derzeitige hohe Papierverbrauch - vor allem im Verpackungsbereich - ist ohne den hohen Altpapiereinsatz nicht tragbar, da die Ressource Holz nicht ausreichend zur Verfügung steht.

Bisher werden grafische Altpapiere und Verpackungsaltpapiere in Deutschland überwiegend gemeinsam erfasst. Es werden auch Altpapiersorten mit Anteilen von grafischen Papierprodukten, wie Druckerzeugnissen, für die Herstellung von Verpackungspapieren eingesetzt. Deshalb wurden von der INTECUS GmbH Dresden und der TU Darmstadt im Auftrag der Papierindustrie die Auswirkungen der rennung der Altpapierkreisläufe für Druckerzeugnisse und Verpackungspapiere in einem Gutachten geprüft (Bilitewski et al. 2011). Eine 100\%ige Trennung ist in der Praxis nicht zu erzielen. Dafür ist die Akzeptanz für weitere Papiertonnen zu gering und die Gefahr von Fehlwürfen zu hoch. Die weitgehend getrennte Erfassung würde zu zusätzlichen Kosten in Höhe von 65-85 Mio. $€$ führen und könnte den Mineralölgehalt im Recyclingpapier nur um ca. 30 \% senken. Die Hersteller von Verpackungen aus Altpapier setzen inzwischen vor allem Altpapiersorten mit einem geringen Anteil an bedruckten Zeitungspapieren ein. Sie haben bereits auf mineralölfreie Druckfarben im Verpackungsdruck umgestellt und testen funktionelle Barrieren. Trotz dieser Maßnahmen hat sich seit 2009 die Belastung kaum geändert (BMELV 2012).

Diese unveränderte Situation ist aus der Sicht des Umweltbundesamtes vor allem darauf zurückzuführen, dass an der wesentlichen Quelle - dem Mineralölgehalt von Zeitungsdruckfarben - nichts geändert wurde. Ungefähr $70.000 \mathrm{t}$ Mineralöl werden insgesamt in den europäischen Papierkreislauf eingetragen und verteilen sich auf die unterschiedlichen Altpapiersorten. In der Verpackungspapier- und Kartonherstellung ist der Altpapiereinsatz besonders hoch. Bei der Herstellung dieser Papiere lässt sich die Druckfarbe allerdings nur zu ca. $70 \%$ unter hohem Aufwand mit ca. 20 \% Rohstoffverlusten entfernen. Durch die Umstellung auf mineralölfreie Farben in allen Offsetdruckverfahren könnte der Eintrag von Mineralöl in den Papierkreislauf sehr effektiv bei vergleichsweise geringen Gesamtkosten vermieden werden. Bisher wurden dazu mehrere Gespräche mit Akteuren der Wertschöpfungsketten geführt. Mehrere Druckversuche verliefen erfolgreich, wie auch die Ergebnisse von Untersuchungen (Zurfluh et al. 2013) veröffentlicht in dieser JVL-Ausgabe bestätigen. Druckfarbenhersteller bekräftigen, dass mineralölfreie Druckfarben lieferbar sind, bislang jedoch kaum Nachfrage besteht. Experten schätzen die Mehrkosten für die Umstellung auf mineralölfreie Druckfarben auf 360 Mio. $€$ (Hotop 2011); umgerechnet ergibt das 1 Cent pro Zeitung. Viele Supermarktketten drucken bereits ihre Kundenzeitungen im Rollenoffset-Heatset-Verfahren mineralölfrei. Die Report Verlagsgesellschaft $\mathrm{mbH}$ in Bocholt ließ fast ihre gesamte Zeitungsauflage im vergangenen Jahr im Rollenoffset-Coldset-Verfahren in der niederländischen Druckerei Janssen Rotadruck mineralölfrei drucken.

Mineralölfreie Druckfarben können einen wichtigen Beitrag für eine umfassende und schadstofffreie Kreislaufwirtschaft leisten. Auch die deutschen Verlagshäuser und die Druckbranche als wesentliche Akteure in der Papierrecyclingkette sind daher aufgerufen, die Ergebnisse der erfolgreichen mineralölfreien Druckversuche einzelner Unternehmen auf die flächendeckende Herstellung von Presseprodukten zu übertragen.

Die Hersteller von Druckerzeugnissen sind bisher nicht dazu verpflichtet, ihre Produkte so zu gestalten, dass sie nach dem Gebrauch für eine schadlose und hochwertige Verwertung geeignet sind, denn für eine solche Verpflichtung bedarf es einer näheren Bestimmung der aus den $\S \S 24$ und 25 zur Produktverantwortung im Kreislaufwirtschaftsgesetz (KrWG) konkret erwachsenden Pflichten durch eine Rechtsverordnung. Positive Beispiele sind die Verpackungsverordnung sowie das BatterieGesetz. Eine Verordnung gemäß KrWG für Druckerzeugnisse könnte hier Abhilfe schaffen.

Bisher setzt der Verordnungsgeber auf das Prinzip der Freiwilligkeit. Mit der freiwilligen Selbstverpflichtung der Arbeitsgemeinschaft Graphischer Papiere (AGRAPA) zur Verwertung Graphischer Altpapiere werden diese Aspekte thematisiert. Alle in dieser Arbeitsgemeinschaft vertretenden Organisationen verpflichten sich: „für Erzeugnisse 
aus Papier, Karton und Pappe durch geeignete Maßnahmen [...] sicherzustellen, dass das hohe $\mathrm{Ma}$ an Kreislaufschließung bei Papier, Karton und Pappe und den daraus hergestellten Produkten sowohl quantitativ als auch qualitativ fortgeschrieben und wo, technisch und wirtschaftlich möglich, noch verbessert wird."

Diese Kernverpflichtung der Selbstverpflichtungserklärung umfasst den gesamten Papierkreislauf und wird durch spezielle Verpflichtungen der einzelnen Unterzeichner spezifiziert. Verleger und die Druckindustrie verpflichten sich insbesondere dazu, soweit wie möglich, nur solche Fertigungsmaterialien und -hilfsmittel (insbesondere Druckfarben und Kleber) sowie Drucktechniken einzusetzen, die eine Verwertung nicht behindern (AGRAPA 1994).

Um dem Leitgedanken der Selbstverpflichtung Rechnung zu tragen, sollten die Mineralöle möglichst schon an der Quelle eliminiert werden, damit diese „eine ordnungsgemäße, schadlose und verarbeitungstechnisch möglichst optimale Verwertung nicht behindern“. Hier sind die Verleger aufgefordert, ihren Beitrag im Rahmen der Selbstverpflichtung zu leisten.

Nach wie vor ist der Durchbruch für eine nachhaltige Lösung der Mineralölproblematik im Sinne einer sauberen Kreislaufwirtschaft nicht in Sicht. Deshalb stellt sich die Frage, ob freiwillige Selbstregulierung der zielführende Weg zu sauberen und ressourcenschonenden Verpackungen aus Altpapier ist oder doch auf ordnungsrechtliche Instrumente zurückgegriffen werden muss, wie sie die Einführung der Produktverantwortung für graphische Papierprodukte im Rahmen einer Verordnung nach KrwG darstellt.

\section{Literatur}

AGRAPA (1994) Selbstverpflichtung der Arbeitsgemeinschaft graphische Papiere (AGRAPA) für eine Rücknahme und Verwertung gebrauchter graphischer Papiere. http://www. gesparec.de/fwselbst.html

BfR (2009) Bundesinstitut für Risikobewertung (BfR). Übergänge von Mineralöl aus Verpackungsmaterialien auf Lebensmittel. Stellungnahme Nr. 008/2010 des BfR vom 09. Dezember 2009. http://www.bfr.bund.de/cm/343/ uebergaenge_von_mineraloel_aus_verpackungsmaterialien _auf_lebensmittel.pdf

Bilitewski B, Wagner J, Kügler T, Schabel S (2011) Putz H-J (2011) Möglichkeiten der getrennten Erfassung und des Recyclings von Zeitungsaltpapier. Studie im Auftrag des Verbandes Deutscher Papierfabriken, Bonn

BMELV (2012) Bundesministeriums für Ernährung, Landwirtschaft und Verbraucherschutz 2012. Ausmaß der Migration unerwünschter Stoffe aus Verpackungsmaterialien aus Altpapier in Lebensmittel. Projekt 2809HS012. http:// download.ble.de/09HS012.pdf

Hotop V (2011) Position der Zeitungsindustrie. Vortrag gehalten auf der BfR-Tagung „Mineralöle in Lebensmittelverpackungen - Entwicklungen und Lösungsansätze“ am 22.09.2011 in Berlin, recherchiert am 22.03.2013 unter http://www.bfr.bund.de/cm/343/positionder-zeitungsindustrie.pdf

Zurfluh M, Grob K, Fink M, Amrein TM (2013) Printing newspaper free of mineral oil: report on a test run. J Verbr Lebensm. doi: 10.1007/s00003-013-0804-5 